\title{
RISCO DE ÚLCERAS DE MEMBROS INFERIORES NOS DIABÉTICOS DE UM AMBULATÓRIO UNIVERSITÁRIO
}

\section{THE RISK OF ULCERATION OF LOWER LIMBS IN DIABETICS OF UNIVERSITY OUTPATIENT CLINIC}

Ana C. Ravazzani1; Ana C. P. Micali2; Daniele de Lemos2; Luiza D. E. Santos2; Maria I. M. Guerra2

\section{RESUMO}

Estudo observacional transversal de 100 pacientes com diabetes tipo 2, sendo 66 homens e 34 mulheres, os quais responderam a um questionário e foram submetidos ao exame físico dos pés, com o objetivo de estratificar o risco de ulceração de membros inferiores em pacientes diabéticos tipo 2 do Ambulatório de Endocrinologia da Santa Casa de Misericórdia de Curitiba, PR.

Descritores: Pé diabético. Complicações crônicas do DM tipo 2. Úlceras de membros inferiores. Doença Arterial Periférica. Neuropatia Periférica.

\section{ABSTRACT}

Observational cross-sectional study of 100 patients with type 2 diabetes, 66 men and 34 women, who answered a questionnaire and underwent a physical examination of the feet, in order to stratify the risk of the ulceration of the lower limbs in type 2 diabetic patients of Endocrinology Outpatient Clinic at Santa Casa de Curitiba-PR.

Keywords: Diabetic foot. Chronic complications of type 2 diabetes. Ulcers of thelower limbs. Peripheral Artery Disease. Peripheralneuropathy.

1 - Professora da disciplina de Endocrinologia da Pontifícia Universidade Católica do Paraná, Curitiba, Paraná.

2 - Graduandas do curso de Medicina da Pontifícia Universidade Católica do Paraná, Curitiba, Paraná.

Contato do Autor / Mail to:

Ana Carolina Pires Micali - carolina_micali@hotmail.com

Avenida Marechal Floriano Peixoto, 2509 - Rebouças - Ambulatório de Endocrinología da Santa Casa de Misericórdia.

CEP 80220-000, Curitiba, Paraná. 


\section{INTRODUÇÃO}

Diabetes Mellitus (DM) é definido como um grupo heterogêneo de distúrbios metabólicos resultantes de defeitos relacionados à ação da insulina, à sua secreção ou a ambas e que apresenta como cerne a hiperglicemia 1 . Estima-se que existam cerca de 387 milhões de pessoas portadoras de DM, das quais $80 \%$ vivem nos países em desenvolvimento e 12 milhões no Brasil1,2. Dentre as complicações tardias do DM, as "neuropatias diabéticas" (ND) são as enfermidades mais comuns a longo prazo, afetando $50 \%$ dos pacientes. As ND são caracterizadas por uma perda progressiva das fibras nervosas ${ }^{1}$, sendo a mais comum delas a polineuropatia diabética sensitivomotora (PND), caracterizada pelo acometimento simétrico e distal, e é causada pelas alterações metabólicas e microvasculares produzidas pela exposição hiperglicêmica crônica ${ }^{3}$.

Há evidências de que as ND e suas sequelas tardias aumentam o risco de amputação e mortalidade ${ }^{4,5}$. Ao alcançar o controle glicêmico ótimo utilizando a terapia intensiva de insulina reduz-se a incidência de PND em pacientes com DM insulinodependente (DMID), como demonstrou 0 estudo Diabetes ControlandComplicationsTrial $($ DCCT) 6 . 0 mesmo é verdadeiro para pacientes com DM 2, como demonstrou o estudo The UK Prospective Diabetes Study (UKPDS). Este estudo também relatou uma prevalência de 13\% de PND ao diagnóstico 7 .

Outra complicação tardia do DM com potencial risco para úlcera de pé diabético é a doença arterial periférica (DAP), que afeta pacientes mais jovens ${ }^{8}$. Sua principal etiologia é a aterosclerose e, na maioria das vezes, coexiste com o acometimento de outros sítios. No paciente diabético, a DAP constitui um fator de risco independente para o desenvolvimento de úlcera de pé, e encontra-se relacionada à evolução desfavorável e ao aumento do risco de amputação?.

Dados do Framingham Heart Study demonstraram que $20 \%$ dos pacientes sintomáticos para DAP apresentam DM, mas essa porcentagem deve ser maior, já que a PND pode mascarar os sintomas de claudicação e outras manifestações da DAP10. Por essa razão, um paciente com DM e DAP pode ser mais suscetível à úlcera ou gangrena do que um paciente sem $\mathrm{DM}^{9}$.

Sabe-se que $10-20 \%$ dos pacientes com PND possuem sintomas que necessitam de tratamento. Dessa forma, a ausência de sintomas não pode ser considerada como ausência de ND11. O diagnóstico clínico da PND baseia-se no exame neurológico dos membros inferiores (MMII). O diagnóstico da DAP também se baseia no exame físico, que deve ser realizado em todo paciente com DM e naqueles que apresentam ulceração, juntamente com o doppler vascular de ondas contínuas, que auxilia na medida do Índice Tornozelo Braço (ITB) ${ }^{9}$.

As complicações crônicas do DM 2 elevam o custo da doença para o Sistema Único de Saúde (SUS) ${ }^{3}$.
No entanto, grandes estudos populacionais sobre a prevalência de PND na população brasileira ainda não foram realizados, e os principais dados epidemiológicos são oriundos de estudos regionais ${ }^{9}$.

O principal objetivo deste estudo é identificar o risco de desenvolvimento de ulceração de membros inferiores em pacientes com DM 2 do ambulatório de Endocrinologia do Hospital Santa Casa de Misericórdia de Curitiba - PR.

\section{METODOLOGIA}

Este estudo foi aprovado pelo Comitê de Ética em Pesquisa (CAAE $n^{\circ}$ 37398314.7.0000.0020 e Parecer $n^{\circ}$ 897.097, de 02 de dezembro de 2014) da Pontifícia Universidade Católica do Paraná (PUCPR). Trata-se de um estudo observacional transversal, cuja população alvo constitui-se dos pacientes do Ambulatório de Endocrinologia da Santa Casa de Misericórdia de Curitiba. Estes foram recrutados de forma aleatória e espontânea, de dezembro de 2014 a setembro de 2015, para responderem a um questionário e serem submetidos ao exame físico dos pés.

A população foi de 100 pacientes com DM tipo 2 (34 homens e 66 mulheres), maiores de 18 anos. Estes assinaram um Termo de Consentimento Livre e Esclarecido (TCLE) sobre os riscos e benefícios de participarem do estudo.

O questionário consistia em perguntas referentes ao tratamento para DM (insulinoterapia ou antidiabéticos orais), às complicações crônicas do DM (neuropatia, nefropatia, retinopatia e doença macrovascular), à presença e ao tratamento de sintomas neuropáticos, e à presença de úlceras ou amputações prévias em MMII.

Quanto aos sintomas neuropáticos, os pacientes foram classificados em Assintomáticos e Sintomáticos Leves, Moderados ou Graves de acordo com o Escore de Sintomas Neuropáticos, e para os pacientes com sintomas dolorosos, foi atribuído um valor de 0 a 10 pela Escala Visual Analógica (EVA) para dor e classificados em dor Leve, Moderada ou Grave ${ }^{1}$.

Em seguida, os participantes foram submetidos ao exame dos pés, para a identificação de patologias não ulcerativas e fatores de risco para ulceração através da inspeção estática dos pés, avaliação dinâmica das mãos (Sinal da Prece) e avaliação do calçado. Na sequência, foram realizados os testes para avaliação de Perda da Sensibilidade Protetora (PSP), Escore de Sinais Neuropáticos e Doença Arterial Periférica (DAP).

Para a avaliação da PSP foram testados: a percepção de pressão (uso do Monofilamento de SemmesWeinstein de $10 \mathrm{~g}$ ), a sensibilidade dolorosa (uso de palito), a sensibilidade vibratória (uso do diapazão de128Hz), a sensibilidade térmica (uso do diapazão), e a avaliação do reflexoAquileu (uso do Martelo de Buck). Foi considerado PSP o exame da percepção de pressão 
alterado em qualquer ponto, associado a pelo menos um dos demais testes citados alterados ${ }^{1}$. Estes dados foram utilizados para cálculo do Escore de Sinais Neurológicos sugerido por Abbott et al. e os sinais foram classificados em Sinais Leves, Moderados ou Graves ${ }^{11}$.

A DAP foi avaliada através da palpação dos pulsos pediosos e tibiais posteriores bilateralmente, através da realização do Índice Tornozelo-Braço (ITB), que consiste na relação entre a medida da pressão sistólica de tornozelo e de braço. 0 teste é realizado com o paciente em decúbito dorsal, com um Doppler manual portátil com transdutor de 10 Mhz e um esfignomanômetro. Afere-se a pressão sistólica (PAS) das artérias tibial posterior e pediosa de ambos os pés e divide-se o maior valor pelo maior valor da PAS das artérias braquiais, aferidas bilateralmente.

Foi considerado o provável diagnóstico de DAP quando houve diminuição ou ausência dos pulsos pedioso ou tibial posterior e/ou valores alterados no $\operatorname{ITB}(<0,9)$. Naqueles pacientes em que não foi possível o cálculo do ITB, apenas a diminuição ou ausência de pulso foram consideradas como provável diagnóstico de DAP.

A classificação de risco do pé diabético utilizada foi preconizada pela Diretriz da Sociedade Brasileira de Diabetes 1 :

- Risco 0 (muito baixo): Ausência de PSP, Ausência de DAP;

Deformidades;

- Risco 1 (baixo): PSP com ou sem

- Risco 2 (moderado): DAP na presença ou ausência de PSP;

- Risco 3 (alto): Úlcera / amputação prévia

O questionário e o exame dos pés seguem o modelo da ficha de Avaliação e Rastreamento de Dor Neuropática, Perda da Sensibilidade Protetora e Doença Arterial Periférica para Atenção Básica a Saúde. Foi adicionada à ficha de cada paciente o último valor da hemoglobina glicada (HbA1C), dos últimos 12 meses. Foi possível adquirir a HbA1C de 78 pacientes da amostra total.

Ao final do exame, os participantes receberam orientações quanto aos cuidadosadequados dos pés para a prevenção de úlceras. Os pacientes sintomáticos ou que apresentavam alguma alteração durante o exame receberam as orientações e encaminhamentos necessários.

Os resultados de variáveis quantitativas foram descritos por médias, medianas, valores mínimos, valores máximos e desvios padrões. Variáveis qualitativas foram descritas por frequências e percentuais. Quanto às variáveis quantitativas, para a comparação de dois grupos, foi utilizado $o$ teste $t$ de Student para amostras independentes ou o teste não paramétrico de MannWhitney. Para a comparação de três grupos foi considerado o modelo de análise da variância (ANOVA) com um fator ou o teste não paramétrico de Kruskal-Wallis.
A condição de normalidade das variáveis foi avaliada pelo teste de Kolmogorov-Smirnov. Em relação às variáveis qualitativas, as comparações foram feitas usando-se o teste exato de Fisher ou o teste de Qui- quadrado. Valores de $p<0,05$ indicaram significância estatística. Os dados foram analisados com o programa computacional IBM SPSS Statistics v.20.

\section{RESULTADOS}

A média de idade dos participantes foi de $63,4 \pm$ 8,4 anos. 0 tempo médio de diagnóstico da doença foi de $12,9 \pm 8,6$ anos. Quanto ao tratamento, 15\% utilizavam apenas insulina, $35 \%$ apenas antidiabéticos orais (ADOs) e metade dos pacientes utilizavam insulina e ADOs combinados. $71 \%$ dos pacientes relataram durante 0 exame já ter diagnóstico de pelo menos uma complicação crônica do DM, sendo a mais prevalente a doença macrovascular, que acometeu $36 \%$ da amostra. O valor médio da última $\mathrm{HbA} 1 \mathrm{C}$ foi de 7,98 \pm 1,9\% (Tabela 1).

Tabela 1: Características gerais da população

\begin{tabular}{l|l}
\hline Características & Amostra \\
\hline Sexo feminino \% & 66 \\
Idade (anos) & $63,4 \pm 8,4$ \\
Tempo de DM (anos) & $12,9 \pm 8,6$ \\
Valor da última HbA1c (\%) & $7,98 \pm 1,91$ \\
Terapia & 35 \\
Uso de ADOs \% & 50 \\
Uso de ADOs e Insulina \% & 15 \\
Uso apenas de Insulina \% & 71 \\
Complicações crônicas \% & 22 \\
Neuropatia \% & 23 \\
Nefropatia \% & 34 \\
Retinopatia \% & 36 \\
Doença Macrovascular \% & \\
\hline
\end{tabular}

analisada:

$\mathrm{Na}$ inspeção dos pés foram encontradas em grande porcentagem dos pacientes várias patologias pré ulcerativas e fatores de risco para úlceras, sendo as mais frequentes: pele seca, calos, calçados inadequados, rachaduras plantares e micoses. Dentre as deformidades, o valgismo e o pé em garra foram as alterações mais frequentes (Tabela 2).

Dos pacientes avaliados, 76 apresentaram queixa de dor ou outro sintoma neuropático, sendo que destes, 20 (26,3\%) apresentaram sintomas leves, 26 $(34,2 \%)$ sintomas moderados e 30 (39,5\%) sintomas graves. Quanto ao Sintoma de Dor, o valor médio na EVA foi de 4,5 (dor moderada). 
Quanto ao Escore de Sinais Neuropáticos, 59,6\% pacientes apresentam-se normais, 25,3\% com comprometimento leve, $9,1 \%$ com comprometimento moderado e $6,1 \%$ com comprometimento grave. A nota média atribuída ao comprometimento destes pacientes foi de 2,5 (Escore de Sinais Leve) (Gráfico 1).

Tabela 2: Achados clínicos na inspeção dos pés e exame da limitação da mobilidade articular.

\begin{tabular}{l|l}
\hline Achados da Inspeçäo & Amostra \% \\
\hline Pele seca & 90,0 \\
Rachadura & 63,0 \\
Calos & 73,0 \\
Calçados inadequados & 59,0 \\
Micose Ungueal & 58,0 \\
Pé em Valgismo & 57,6 \\
Pé em Garra & 42,4 \\
Micose Interdigital & 42,0 \\
Limitação da Mobilidade Articular & 40,4 \\
Fissuras & 27,0 \\
Pés Cavos & 18,2 \\
Pés Charcot & 14,1 \\
Vasos dilatados dorsais & 3,0 \\
Palidez & 1,0 \\
Cianose & 0 \\
\hline & \\
\hline
\end{tabular}

Quanto à classificação do Escore de Sinais Neuropáticos, houve diferença estatisticamente significativa entre os grupos em relação ao tempo de DM $(p=0,025)$, sendo que os pacientes classificados como graves apresentam maior tempo médio de doença, bem como a presença de complicações crônicas, em que se verificou maior prevalência destas entre os pacientes com classificação moderada e grave $(p=0,021)$.

A PSP esteve presente em $26 \%$ dos participantes. Houve diferença significativa entre os grupos com ou sem PSP em relação ao tempo de DM $(p=0,049)$, sendo que os pacientes com PSP têm um tempo médio superior de doença do que aqueles que sem PSP.

Houve correlação significativa entre a presença de PSP e os Escores de Sinais e Sintomas Neuropáticos, de modo que os pacientes com PSP apresentam maior valor para os dois parâmetros (Tabela 3).

Dos participantes, 63\% apresentaram-se com risco de DAP, 37\% apresentaram-se sem DAP. Houve correlação significativa do risco de DAP com idade $(p=0,012)$ e tempo de $D M(p=0,025)$ e à pontuação no Escore de Sinais Neuropáticos $(p=0,011)$.

Durante o exame dos pés, 6 pacientes apresentaram-se com úlcera em MMII ativa. No entanto,
15 participantes relataram úlcera prévia. Além disso, 2 pacientes avaliados apresentaram amputação de MMII.

$\mathrm{Na}$ estratificação de risco de ulceração de MMII, 50 participantes apresentaram-se com risco $0 ; 12$ participantes com risco 1; 23 participantes com risco 2 e 15 pacientes com risco 3 (Gráfico 2).

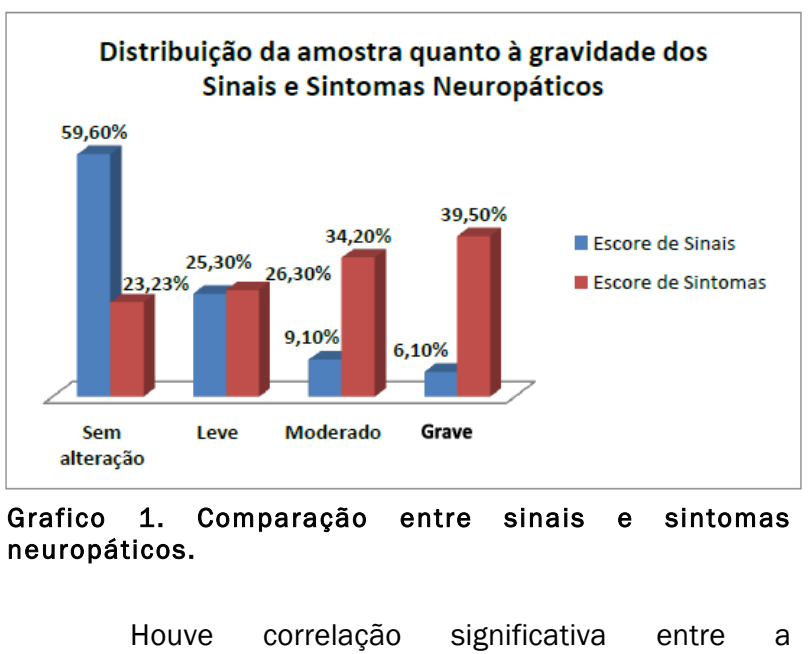
Classificação de Risco e o tempo de DM ( $p=0,009)$, sendo que os pacientes com risco maior apresentam maior tempo médio de DM. Esta relação também pode ser encontrada na presença de complicações crônicas do DM em conjunto $(p=0,003)$, sendo que pacientes que não apresentam complicações crônicas estão predominantemente no risco $0(75,86 \%)$, e pacientes com complicações crônicas estão distribuídos entre o risco 0 $(39,44 \%)$, risco $1(11,27 \%)$, risco $2(29,58 \%)$ e risco 3 $(19,72 \%)$.

Pode-se verificar também diferença significativa da Classificação de Risco com o risco de DAP e a presença de PSP $(p<0,001)$ (Tabela 4). Não houve diferença na Classificação de Risco quanto às demais variáveis.

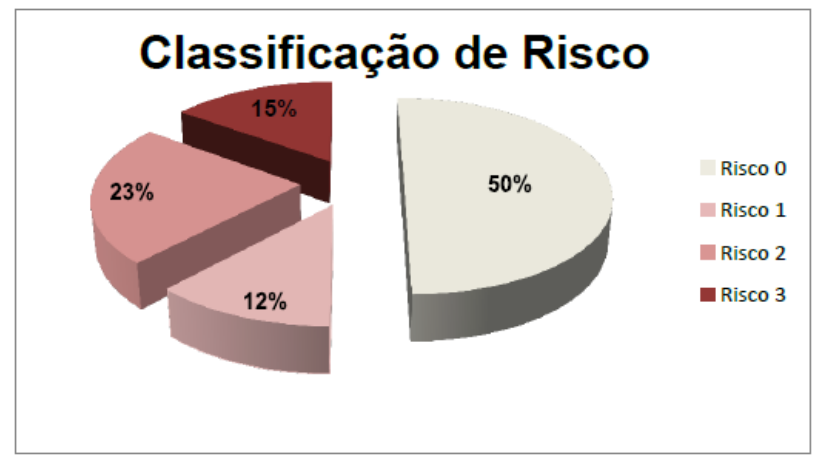

Grafico 2. Classificação de risco da população analisada.

\section{DISCUSSÃO}

As características da população analisada foram compativeis com a maioria dos estudos previamente revisados $12-15$ (Tabela 5). 
Tabela 3: Comparação entre a PSP e os Escores de Sinais e Sintomas Neuropáticos

\begin{tabular}{lcccccccc}
\hline & PSP & $\mathbf{n}$ & Média & Mediana & Mínimo & Máximo & $\begin{array}{c}\text { Desvio } \\
\text { padrão }\end{array}$ & $\begin{array}{c}\text { Valor de } \\
\mathbf{p}^{*}\end{array}$ \\
\hline $\begin{array}{l}\text { Escore de } \\
\text { sintomas } \\
\text { neuropáticos }\end{array}$ & Sim & 26 & 5,6 & 6,5 & 0 & 9 & 3,0 & \\
& Não & 74 & 4,1 & 4 & 0 & 9 & 2,9 & 0,013 \\
$\begin{array}{l}\text { Escore de sinais } \\
\text { neuropáticos }\end{array}$ & Sim & 26 & 4,6 & 4 & 1 & 10 & 3,1 & \\
\hline & Não & 73 & 1,7 & 0 & 0 & 9 & 2,3 & $<0,001$ \\
\hline
\end{tabular}

Conforme visto em outros estudos, a amostra foi composta predominantemente por mulheres (66\%), visto que as mulheres comparecem em maior número às consultas para acompanhamento do $\mathrm{DM}^{1}$. 0 tempo médio do diagnóstico do DM foi de 12,9 anos, característico depopulações diabéticas em serviços terciários, comotambém mostrado em outros estudos brasileiros ${ }^{13,15}$.

Tabela 4: Comparação entre a Classificação de Risco e o Risco de DAP e presença de PSP

\begin{tabular}{|c|c|c|c|c|}
\hline \multirow{2}{*}{ Classificação de risco } & \multicolumn{2}{|c|}{ Risco de DAP } & \multicolumn{2}{|l|}{ PSP } \\
\hline & Sim & Não & Sim & Não \\
\hline 0 & $36.51 \%$ & $72.97 \%$ & $0.00 \%$ & $67.57 \%$ \\
\hline 1 & $6.35 \%$ & $21.62 \%$ & $46.15 \%$ & $0.00 \%$ \\
\hline 2 & $36.51 \%$ & $0.00 \%$ & $30.77 \%$ & $20.27 \%$ \\
\hline 3 & $20.63 \%$ & $5.41 \%$ & $23.08 \%$ & $12.16 \%$ \\
\hline
\end{tabular}

Constatou-se que o valor médio de $\mathrm{HbA} 1 \mathrm{C}$ dos pacientes foi de $7,98 \%$, o que demonstra que a maioria apresenta-se fora da meta preconizada pela Sociedade Brasileira de Diabetes $(<7 \%)^{1}$. No entanto, não foi verificada relação significativa entre o valor da última HbA1C e a classificação de risco ou a presença de DAP.

Neste estudo $26 \%$ dos pacientes apresentavamse com PSP, enquanto a prevalência encontrada na literatura variou entre $21,3 \%$ à $36 \%$, sendo que, em geral, os dados nacionais tendem a ser superiores a 30\%11-14.

Apesar de 76,8\% dos pacientes avaliados apresentaram-se sintomáticos, dentre eles, 39,5\% com sintomas graves, a PSP foi constatada em apenas $26 \%$. Esses dados se contrapõem com uma coorte realizada por Abbott et al., no Reino Unido, com 15.692 participantes, em que $48 \%$ dos pacientes apresentavam sintomas neuropáticos, dentre eles $16 \%$ sintomas graves, mas a PSP foi encontrada em $65 \% 11$.

$\mathrm{Na}$ amostra atual, 59,6\% dos pacientes não apresentaram comprometimento neuronal quando avaliados pelo Escore de Sinais Neuropáticos. Esses dados são compatíveis com o estudo de Santos e com a coorte já citada do Reino Unido11,12.

Alguns estudos mostram que a hiperglicemia persistente pode gerar alterações vasculares e metabólicas nos componentes neurais ${ }^{15}$. Porém, neste estudo não houve correlação entre a HbA1C e a PND. 0 tempo de DM, por sua vez, pode ser relacionado à gravidade do comprometimento neurológico pelo Escore

Tabela 5: Comparação entre a amostra do estudo atual com a de quatro estudos brasileiro e um estudo do Reino Unido

\begin{tabular}{ccccccc}
\hline Variáveis & RoLIM et al. & $\begin{array}{c}\text { MOREIRA } \\
\text { et al. }\end{array}$ & TRES et al. & $\begin{array}{c}\text { SCHEFFEL } \\
\text { et al. }\end{array}$ & $\begin{array}{c}\text { ABBOTT et } \\
\text { al. }\end{array}$ & Estudo atual \\
\hline N & 94 & 57 & 340 & 927 & 15.692 & 100 \\
\hline $\begin{array}{c}\text { Idade } \\
\text { (anos) }\end{array}$ & $56,9 \pm 10,3$ & $53,1 \pm 7,0$ & $57.8 \pm 11$ & $59 \pm 10$ & $61.4 \pm 14.0$ & $63,4 \pm 8,4$ \\
\hline $\begin{array}{c}\text { DM 2 (\%) } \\
\text { Tempo de }\end{array}$ & 78,7 & 100 & 100 & 100 & 91,4 & 100 \\
DM (anos) & $10,4 \pm 6,8$ & - & $8 \pm 7$ & 11 & $5(2-10)$ & $12,9 \pm 8,6$ \\
\hline $\begin{array}{c}\text { HbA1c } \\
\text { Genero }\end{array}$ & $8,4 \pm 2,1$ & $7,8 \pm 2,2$ & $8.1 \pm 2.09$ & $6,8 \pm 1,9$ & - & $7,98 \pm 1,91$ \\
feminino \\
(\%)
\end{tabular}


de Sinais Neuropáticos.

Em relação à DAP, o estudo de Spichler et al. demonstrou que o sexo masculino e faixas etárias de 6569 e 55-79 anos apresentavam maiores índices de DAP em pacientes com e sem $\mathrm{DM}^{13}$. Outros estudos demonstram que a prevalência de DAP é maior em mulheres com DM14.

No presente estudo, foi encontrada correlação entre DAP e a idade dos pacientes, mas não quanto ao sexo. No estudo de Santos et al., que avaliou fatores relacionados à amputação do pé diabético, foi encontrado maior número de amputações nos pacientes com mais de 60 anos $^{16}$. Conforme o presente estudo, 63\% dos pacientes diabéticos apresentaram alteração à palpação do pulso, e destes, 52,3\% apresentaram ITB alterado e por isso preencheram os critérios para DAP. Porém, é importante ressaltar que o exame físico dos pés (em especial a palpação de pulso) pode apresentar valor limitado no diagnóstico de DAP em pacientes diabéticos, sendo necessária avaliação por meio do Doppler1,17,18. No entanto, a revisão sistemática do InternationalWorkingGroupontheDiabeticFoot demonstrou que a coexistênciade DAP e neuropatia em pacientes diabéticos é muito frequente, sendo que o emprego do ITBpara diagnóstico de DAP no diabetes tem valor limitado, devido a maior associação com calcificação arterial (arteriosclerose de Monckeberg)17,19. Portanto, seria interessante utilizar outros exames, como a medida da pressão do pododáctilo e cálculo do Índice Dedo Braquial (IDB), para confirmar a real prevalência da associação entre DAP e neuropatia $8,17,18$.

O escore de risco foi maior para pacientes com mais tempo de DM. Esse fato já era esperado, pois uma duração de diabetes superior a 10 anos está diretamente relacionada com aumento do risco ${ }^{1}$.

Neste estudo, a presença de complicações crônicas foi diretamente relacionada ao risco de ulceração de MMII, como também mostram outros estudos, em que pacientes com complicações micro e macrovasculares do DM apresentavam mais neuropatia sensitiva distal, que é fator de risco importante para úlcera e amputação13,15.

\section{CONCLUSÃO}

$\mathrm{Na}$ avaliação de 100 pacientes da população diabética do Ambulatório de Endocrinologia da Santa Casa de Curitiba, encontrou-se cerca de $15 \%$ dos pacientes classificados como risco 3 na estratificação de risco para ulceração/amputação de membros inferiores, o que significa uma população de alto risco.

Foi encontrada uma correlação entre a classificação de risco do pé diabético e as variáveis tempo médio de DM e complicações crônicas do DM, o que sugere a necessidade de acompanhamento especial desta população.

Por fim, o exame sistemático dos pés faz-se necessário nas consultas de endocrinologia para quantificar o risco de úlceras/amputações de membros inferiores em pacientes diabéticos sintomáticos ou não, a fim de melhorar as estratégias de tratamento e prevenção desta complicação.

\section{CONFLITOS DE INTERESSE}

Não houve conflitos de interesse.

\section{AGRADECIMENTOS}

Os autores desta pesquisa agradecem ao colega Daniel Renal Duarte Alves Lima pelas colaborações prestadas a este trabalho.

\section{REFERÊNCIAS}

1. Diabetes SB do. Diretrizes da Sociedade Brasileira de Diabetes 2014-2015. Sociedade Brasileira do Diabetes. 2015;

2. Mena NA, Sea EA, Lucia S. IDF Diabetes Atlas 6th edn. 2014 update. International Diabetes Federation. 2014;

3. PEDROSA HC et au. Consenso Internacional sobre Pé Diabético. Secretaria de Estado de Saúde do Distrito Federal. 2001. p. 100.

4. Aal JUVANB, Effcoate WIJ. Mortality Association with Acute Charcot Foot and Neuropathic Foot Ulceration. Diabetes Care. 2010;33(5):3-6.

5. Study H. History of Foot Ulcer Increases Mortality. Diabetes Care. 2009;32(12).

6. The Diabetes Control and Complications. Trial Reserch Group. The effect of intensive treatment of diabetes on the development and progression of long-term complications in insulin-dependent diabetes mellitus. N Engl J Med. 1993;

7. UK Prospective Diabetes Study (UKPDS) Group. Intensive blood-glucose control with sulphonylureas or insulin compared with conventional treatment and risk of complications in patients with type 2 diabetes (UKPDS 33). Lancet. 1998;

8. Boulton AJM, Armstrong DG, Albert SF, Frykberg RG, Hellman R, Kirkman MS, et al.

9. Comprehensive foot examination and risk assessment. Diabetes Care. 2008;31(8):1679-85.

10. Pedrosa HC VLB. Neuropatia e Pé Diabético. 1a edição. São Paulo: AC FARMACÊUTICA; 2014.

11. The WIS, Peripheral IOF, In AD. Epidemiology and Impact of Peripheral Arterial Disease in.

12. Am Diabetes Assoc [Internet]. 2003;26(12):3333-41. Available from: http://www.ncbi.nlm.nih.gov/pubmed/14633825

13. Abbott $C$ a., Malik $R$ a., van Ross ERE, Kulkarni J, Boulton a. JM. Prevalence and Characteristics of Painful Diabetic Neuropathy in a Large CommunityBased DiabeticPopulation in the U.K. Diabetes Care 
[Internet]. 2011;34(10):2220-4. Available from: http://care.diabetesjournals.org/cgi/doi/10.2337/dc 11-1108

14. Tres GS, Lisbôa HRK, Syllos R, Canani LH, Gross JL. Prevalence and characteristics of diabetic polyneuropathy in Passo Fundo, South of Brazil. Arq Bras EndocrinolMetabol. 2007;51:987-92.

15. Rolim LC, Sá JR De, Chacra AR, Dib SA. Heterogeneidade clínica e coexistência das neuropatias diabéticas: diferenças e semelhanças entre diabetes melito tipo 1 e 2. Arq Bras EndocrinolMetabol. 2009;53(7).

16. Alberto $C$, Isabel $M$, Venâncio L. Pé diabético e avaliação do risco de ulceração. Revista de Enfermagem. 2014;153-61.

17. R. J. Hinchliffe1, J. R. W. Brownrigg1, J. Apelqvist2, E. J. Boyko3, R. Fitridge4, J. L. Mills5 JR, C. P. Shearman7, R. E. Zierler8 NCS on behalf of the IWG on the D, (IWGDF) F. IWGDF Guidance on the diagnosis, prognosis and management of peripheral artery disease in patients with foot ulcers in diabetes. 2015;1-18.

18. Dolan Nancy C, Liu K, Criqui Michael H, Greenland P, Guralnik Jack M, Chan C, et al.

19. Peripheral artery disease, diabetes, and reduced lower extremity functioning. Diabetes Care. 2002;25(1):113-20.

20. Brownrigg JRW, Apelqvist J, Bakker K, Schaper NC, Hinchliffe RJ. Evidence-based management of PAD \& the diabetic foot. Eur J VascEndovascSurg [Internet]. Elsevier Ltd; 2013;45(6):673-81. Available from: http://dx.doi.org/10.1016/j.ejvs.2013.02.014 